Network Working Group

Request for Comments: 4415

Category: Standards Track
R. Brandner

Siemens AG

L. Conroy

Siemens Roke Manor Research

R. Stastny

Oefeg

February 2006

\title{
IANA Registration for Enumservice Voice
}

Status of This Memo

This document specifies an Internet standards track protocol for the Internet community, and requests discussion and suggestions for improvements. Please refer to the current edition of the "Internet Official Protocol Standards" (STD 1) for the standardization state and status of this protocol. Distribution of this memo is unlimited.

Copyright Notice

Copyright (C) The Internet Society (2006).

Abstract

This document registers the Enumservice "voice" (which has a defined subtype "tel"), as per the IANA registration process defined in the ENUM specification RFC 3761. This service indicates that the contact held in the generated Uniform Resource Identifier (URI) can be used to initiate an interactive voice (audio) call.

Table of Contents

1. Introduction . . . . . . . . . . . . . . . . . . . . . 2

2. Terminology . . . . . . . . . . . . . . . . . . . . . 3

3. Voice Service Registration . . . . . . . . . . . . . . . . . 3

4. Example of voice:tel Enumservice . . . . • . • . • . . • . . . 4

5. Security Considerations . . . . . . . . . . . . . . . . . . 4

6. IANA Considerations . . . . . . . . . . . . . . . . . . 5

7. References . • . . . . . . • . . . . . . . • . . . . . 6

7.1. Normative References . • . . . . . . . . . . . . 6

7.2. Informative References . . . . . . . . . . . . . . 6 


\section{Introduction}

ENUM (E.164 Number Mapping, RFC 3761 [1]) is a system that transforms E.164 numbers [2] into domain names and then uses DNS (RFC 1034 [3]) features such as delegation through NS records, and the use of Naming Authority Pointer (NAPTR) records, to look up the communication services available for a specific domain name.

This document registers an Enumservice according to the guidelines given in RFC 3761 to be used for provisioning in the services field of a NAPTR [4] resource record to indicate what class of functionality a given endpoint offers. The registration is defined within the Dynamic Delegation Discovery System (DDDS, [5] [6] [4] [7] [8]) hierarchy, for use with the "E2U" DDDS application defined in RFC 3761 .

Enumservices have a type and subtype. This latter is optional, as it may be implicit in the service type. The type defines the kind of communication session that can be initiated using the contact indicated by the URI generated by the enclosing NAPTR. In telecommunications engineering terms, it reflects the "teleservice".

The subtype defines the subsystem that is to be used to initiate the communication session. Note that the subtype definition is usually associated with the URI scheme that is to be used.

Both the type and subtype (where present) must be supported for the NAPTR to be used by a potential correspondent.

There are a number of DDDS applications in addition to ENUM (for example, see [7] and [8]). However, an Enumservice indication operates only within the context of the "E2U" (ENUM) DDDS Application.

Whilst the protocol elements that make up ENUM are defined in the above documents and in this one, further examples of the use to which these may be put are given in other documents, for example, in ETSI TS 102172 [11].

This document registers the Enumservice "voice" (which has a defined subtype "tel"), as per the IANA registration process defined in the ENUM specification RFC 3761. This service indicates that the contact held in the generated URI can be used to initiate an interactive voice (audio) call. 


\section{Terminology}

The key words "MUST", "MUST NOT", "REQUIRED", "SHALL", "SHALL NOT", "SHOULD", "SHOULD NOT", "RECOMMENDED", "MAY", and "OPTIONAL" in this document are to be interpreted as described in BCP 14, RFC 2119 [9].

3. Voice Service Registration

Enumservice Name: "voice"

Enumservice Type: "voice"

Enumservice Subtype: "tel"

URI Scheme: 'tel:'

Functional Specification:

The kind of communication indicated by this Enumservice is "Interactive Voice". From a protocol perspective, this communication is expected to involve bidirectional media streams carrying audio data.

A client may imply that the person controlling population of a NAPTR holding this Enumservice indicates his capability to engage in an interactive voice session when contacted using the URI generated by this NAPTR.

Security Considerations:

See Section 5 .

Intended Usage: COMMON

Authors:

Rudolf Brandner, Lawrence Conroy, and Richard Stastny (for author contact detail, see Authors' Addresses section)

Any other information the author deems interesting:

This Enumservice indicates that the person responsible for the NAPTR is accessible via the Public Switched Telephone Network (PSTN) or Public Land Mobile Network (PLMN) using the value of the generated URI.

The kind of subsystem required to initiate a Voice Enumservice with this subtype is a "Dialer". This is a subsystem that either 
provides a local connection to the PSTN or PLMN or provides an indirect connection to those networks. The subsystem will use the telephone number held in the generated URI to place a voice call. The voice call is placed to a network that uses E.164 numbers to route calls to an appropriate destination.

Note that the PSTN/PLMN connection may be indirect. The end user receiving this NAPTR may have a relationship with a communications Service Provider that accepts call initiation requests from that subsystem using an IP-based protocol such as SIP or H.323, and places the call to the PSTN using a remote gateway service. In this case, the provider either may accept requests using "tel:" URIs or has a defined mechanism to convert "tel:" URI values into a "protocol-native" form.

The "tel:" URI value SHOULD be fully qualified (using the "global phone number" form of RFC 3966 [10]). A "local phone number" as defined in that document SHOULD NOT be used unless the controller of the zone in which the NAPTR appears is sure that it can be distinguished unambiguously by all clients that can access the resource record and that a call from their network access points can be routed to that destination.

4. Example of voice:tel Enumservice

The following is an example of the use of the Enumservice registered by this document in a NAPTR resource record.

\$ORIGIN 0.6.9.2.3.6.1.4.4.e164.arpa. 3.8.0 NAPTR 10100 "u" "E2U+voice:tel" "!^.*\$!tel: +441414960000!" .

5. Security Considerations

DNS, as used by ENUM, is a global, distributed database. Thus, any information stored there is visible to anyone anonymously. Whilst this is not qualitatively different from publication in a telephone directory, it does open the data subjects to having "their" information collected automatically without any indication that this has been done or by whom.

Such data harvesting by third parties is often used to generate lists of targets for unrequested information; in short, they are used to address "spam". Anyone who uses a Web-archived mailing list is aware that the volume of "spam" email sent increases when he or she posts to the mailing list; publication of a telephone number in ENUM is no different, and may be used for attempts to send "junk faxes" or "junk sMS", for example. 
Many mailing list users have more than one email address and use "sacrificial" email accounts when posting to such lists to help filter out unrequested emails sent to them. This is not so easy with published telephone numbers; the PSTN E.164 number assignment process is much more involved and usually a single E.164 number (or a fixed range of numbers) is associated with each PSTN access. Thus, providing a "sacrificial" phone number in any publication is not possible.

Due to the implications of publishing data on a globally accessible database, as a principle the data subjects MUST give their explicit informed consent to data being published in ENUM.

In addition, they should be made aware that, due to storage of such data during harvesting by third parties, removal of the data from publication will not remove any copies that have been taken; in effect, any publication may be permanent.

However, regulations in many regions will require that the data subjects can at any time request that the data be removed from publication and that their consent for its publication be explicitly confirmed at regular intervals.

When placing a voice call via the PSTN (or from the Public Land Mobile Network), the sender may be charged for this action. In both kinds of networks, calling some numbers is more expensive than sending to others; both kinds of networks have "premium rate" services that can be charged at a rate considerably more than a "normal" call. As such, it is important that end users be asked to confirm placing the call and that the destination number be presented to them. It is the originating user's choice whether or not to place a call to this destination number, but the originating user SHOULD be shown the destination number so that he or she can make this decision.

In addition to the specific security considerations given above, all security considerations given in RFC 3761 apply, as well as the DNS-specific threats covered in RFC 3833 [12].

6. IANA Considerations

The IANA has registered the Enumservice "voice" with a single subtype "tel" according to the framework defined in RFC 3761. The current document defines this Enumservice and the expected behaviour of clients. 
7. References

7.1. Normative References

[1] Faltstrom, P. and M. Mealling, "The E.164 to Uniform Resource Identifiers (URI) Dynamic Delegation Discovery System (DDDS) Application (ENUM)", RFC 3761, April 2004.

[2] ITU-T, "The International Public Telecommunication Number Plan", Recommendation E.164, May 1997.

[3] Mockapetris, P., "DOMAIN NAMES - CONCEPTS AND FACILITIES", RFC 1034, November 1987.

[4] Mealling, M., "Dynamic Delegation Discovery System (DDDS) Part Three: The Domain Name System (DNS) Database", RFC 3403, October 2002 .

[5] Mealling, M., "Dynamic Delegation Discovery System (DDDS) Part One: The Comprehensive DDDS", RFC 3401, October 2002.

[6] Mealling, M., "Dynamic Delegation Discovery System (DDDS) Part Two: The Algorithm", RFC 3402, October 2002.

[7] Mealling, M., "Dynamic Delegation Discovery System (DDDS) Part Four: The Uniform Resource Identifiers (URI)", RFC 3404, October 2002 .

[8] Mealling, M., "Dynamic Delegation Discovery System (DDDS) Part Five: URI.ARPA Assignment Procedures", RFC 3405, October 2002.

[9] Bradner, S., "Key words for use in RFCs to Indicate Requirement Levels", RFC 2119, BCP 14, March 1997.

[10] Schulzrinne, H., "The tel URI for Telephone Numbers", RFC 3966, December 2004 .

\subsection{Informative References}

[11] ETSI, "Minimum Requirements for Interoperability of ENUM Implementations", ETSI TS 102 172, January 2005.

[12] Atkins, D. and R. Austein, "Threat Analysis of the Domain Name System (DNS)", RFC 3833, August 2004 . 


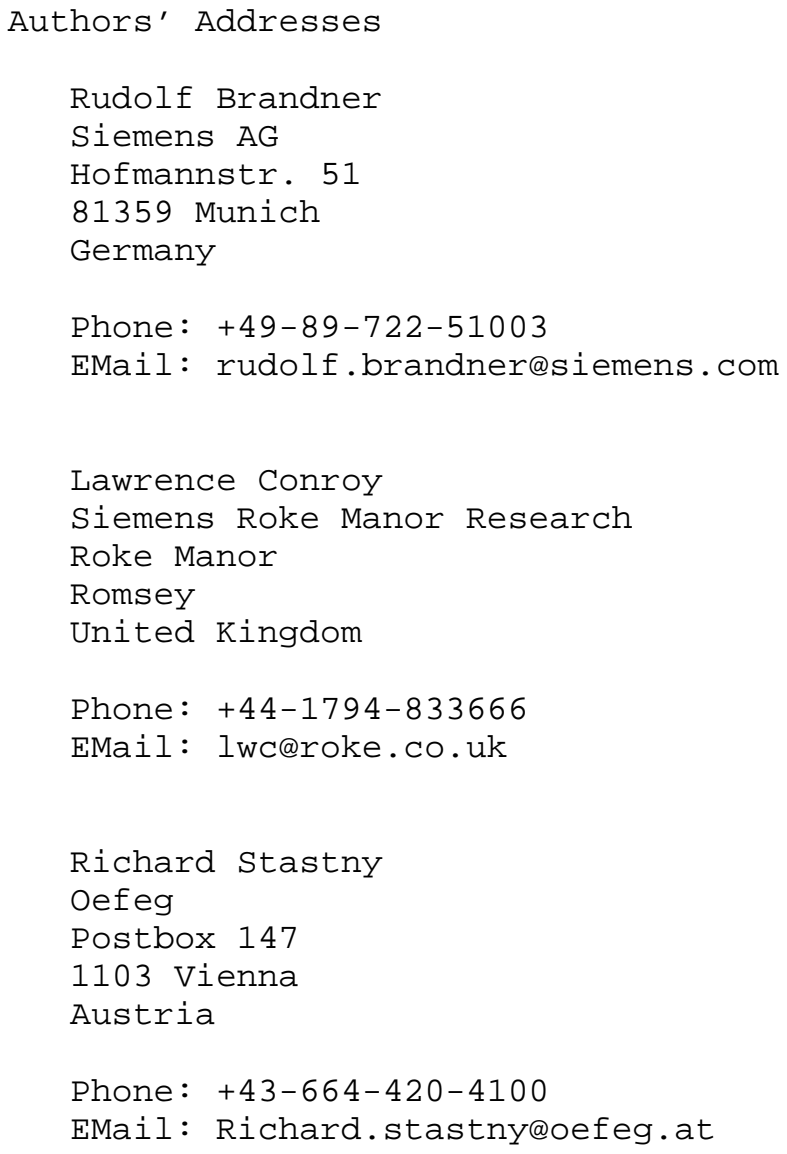


Full Copyright statement

Copyright (C) The Internet Society (2006).

This document is subject to the rights, licenses and restrictions contained in BCP 78, and except as set forth therein, the authors retain all their rights.

This document and the information contained herein are provided on an "AS IS" basis and THE CONTRIBUTOR, THE ORGANIZATION HE/SHE REPRESENTS OR IS SPONSORED BY (IF ANY), THE INTERNET SOCIETY AND THE INTERNET ENGINEERING TASK FORCE DISCLAIM ALL WARRANTIES, EXPRESS OR IMPLIED, INCLUDING BUT NOT LIMITED TO ANY WARRANTY THAT THE USE OF THE INFORMATION HEREIN WILL NOT INFRINGE ANY RIGHTS OR ANY IMPLIED WARRANTIES OF MERCHANTABILITY OR FITNESS FOR A PARTICULAR PURPOSE.

Intellectual Property

The IETF takes no position regarding the validity or scope of any Intellectual Property Rights or other rights that might be claimed to pertain to the implementation or use of the technology described in this document or the extent to which any license under such rights might or might not be available; nor does it represent that it has made any independent effort to identify any such rights. Information on the procedures with respect to rights in RFC documents can be found in BCP 78 and BCP 79 .

Copies of IPR disclosures made to the IETF Secretariat and any assurances of licenses to be made available, or the result of an attempt made to obtain a general license or permission for the use of such proprietary rights by implementers or users of this specification can be obtained from the IETF on-line IPR repository at http://www.ietf.org/ipr.

The IETF invites any interested party to bring to its attention any copyrights, patents or patent applications, or other proprietary rights that may cover technology that may be required to implement this standard. Please address the information to the IETF at ietf-ipreietf.org.

Acknowledgement

Funding for the RFC Editor function is provided by the IETF Administrative Support Activity (IASA). 